\title{
Employment of resonators for resolution enhancement in plasmonic refractive index sensors
}

\author{
Hamid Abbasi \\ University of Mazandaran,Iran.
}

\begin{abstract}
In this paper, a plasmonic refractive index sensor based on metal insulated metal waveguide (MIM) with three rings and a resonant cavity is first proposed and numerically evaluated. Next, we add four teeth to the sensor structure. We study and simulate the resonant wavelengths and refractive index of resonators using the time difference finite difference method. Given that the sensor and the conduction characteristics of plasmonic waves are influenced by the structure parameters, Therefore, by changing the refractive index and changing the dimensions and coordinates of the cavity and rims, we can change the passage coefficient in the resonant modes and measure the sensor performance. As a result, we obtain the sensitivity coefficient, the figure of merit (FOM) and the quality factor $\mathrm{Q}$ of the sensor. We modulate the resonance wavelength FWHM using the generated modes and reach a sensitivity of $2166 \mathrm{~nm} /$ RIU. These plasmonic sensors with a simple framework and balanced performance and high optical resolution can be used to measure the refractive index in the medical, chemical and food industries.
\end{abstract}

Keywords: plasmonics; Surface plasmon polaritons; Metal-Insulator-Metal; refractive index sensor.

\section{Introduction}

The attractive feature of surface plasmon polarites (SPP), ie the restriction of light in nano-dimensions, has caused this phenomenon to be widely studied and this feature can be used to build many plasmonic structures such as filters [1], multiple optical instruments [2], absorbers [3], splitters [4] and sensors [513]. Also metal-insulated-metal plasmonic waveguide (MIM) due to its ability to limit light in a small area and its compatibility with electronic operating systems is widely used in the design of many plasmonic devices. In addition, having a simple design method of these structures has made them one of the most desirable and popular structures. As a result, a variety of MIM plasmonic devices have been designed and implemented [14,15]. Many criteria can be used to design and implement refractive index sensors, one of which is the calculation of good sensitivity (S) and figure of merit (FOM) [1624]. For this reason, researchers have made great efforts to improve the sensitivity of MIM plasmonic sensors, but longer wavelengths always suffer from wider FWHM, which reduces the figure of merit (FOM) and sensitivity coefficient. Recently, to enhance the performance of plasmonic sensors, various approaches have been used, such as Fano resonance with dark and light resonance interference, which results from structural symmetry failure or resonance interference, but such resonance is unstable and can be easily broken due to phase or mode mismatch. In this study, arrays of metal-dielectric-metal (MIM) plasmonic waveguides and resonators are designed, simulated and analyzed to design and fabricate plasmonic sensors. The two-dimensional finite difference time domain (FDTD) method is used to simulate the sensor. Boundary conditions for all directions are selected as the perfectly matched layer (PML). To simulate FDTD, a uniform mesh size of $8 \mathrm{~nm}$ was used.

\section{Structural model and theory analysis}

As shown in Fig.1, two MIM waveguides coupled with three rings and a cavity are proposed. The waveguides are etched on the silver surface. As shown in $2 \mathrm{D}$ picture, the grey and white areas represent silver and air, respectively. The permittivity of air is set as $\varepsilon_{\mathrm{i}}=1$, as for silver $(\mathrm{Ag})$ the Drude model is utilized as follows [1]:

$\varepsilon(\omega)=\varepsilon_{\infty}-\omega_{\mathrm{p}}^{2} / \omega^{2}+\mathrm{i} \Upsilon \omega$ 
Here $\varepsilon_{\infty}=1$ gives the medium constant for the infinite frequency, $\omega p=1.37 \times 1016$ refers to bulk frequency for plasma, $\gamma=3.21 \times 1013$ means damping frequency for electron oscillation, and $\omega$ shows incident light angular frequency. To describe metal and plasmonic waveguides in sensors, the Drude model is used because the Drude model can be easily integrated into time-difference finite difference simulations.

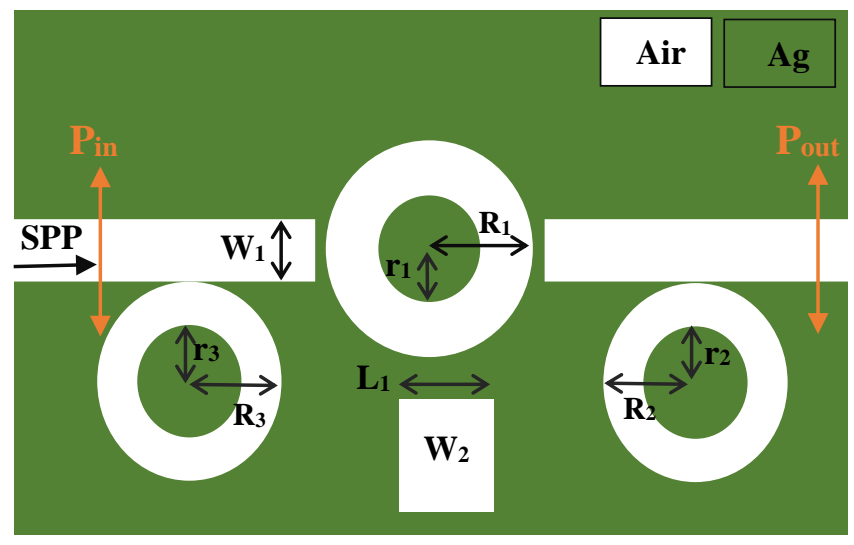

Fig.1. Two-dimensional image of a plasmonic sensor

The height of the two waveguides is $\mathrm{w}_{1}=50 \mathrm{~nm}$. The cavity has a height of $\mathrm{w}_{2}=130 \mathrm{~nm}$ and a length of $L_{1}=92 \mathrm{~nm}$. The middle ring has an inner radius of $r_{1}=90 \mathrm{~nm}$ and an outer radius of $R_{1}=133 \mathrm{~nm}$ and the two side rims have an inner radius of $r_{3}=r_{2}=91 \mathrm{~nm}$ and an outer radius of $R_{3}=R_{2}=126$ $\mathrm{nm}$, respectively. $\mathrm{P}_{\mathrm{in}}$ and $\mathrm{P}_{\text {out }}$ are the monitors for measuring the input wave and the output wave, respectively. These two monitors are computed by merging the natural component of the Poin Ting vector along the blurred lines. As a result, the wave transmission is calculated by the relation:

$\mathrm{T}=\mathrm{P}_{\text {out }} / \mathrm{P}_{\text {in }}$

Surface plasmon polarites (SPP) TM mode, when attached to the MIM structure, can propagate at the interface level in the waveguide. Compared to the excited wavelength, the width of the bass waveguide is much smaller, so only the TM base mode can only participate in the sensor simulation. The input wave starts moving from the left and after passing through the rings and cavities, it goes to the output waveguide. The silicon substrate is made of silver and the reason for this is to strengthen the performance of the refractive index [25]. We also use the following equations to calculate the TM wave scattering relationship [15]:

$\frac{\varepsilon_{\mathrm{i}} \mathrm{p}}{\varepsilon_{\mathrm{m}} k}=\frac{1-e^{\mathrm{kw}}}{1+e^{\mathrm{kw}}}$

$p=k_{0} \sqrt{\left(\frac{\beta_{s p}}{k_{0}}\right)^{2}-\varepsilon_{m}}$

$$
k=k_{0} \sqrt{\left(\frac{\beta_{s p}}{k_{0}}\right)^{2}-\varepsilon_{i}}
$$

$$
\beta_{s p}=\mathrm{n}_{e f f} k_{0}=\mathrm{n}_{\text {eff }} \frac{2 \pi}{\lambda}
$$

Here $\omega$ refer to the width of bus waveguide, $\lambda$ shows incident light wavelength in vacuum, $\varepsilon_{\mathrm{i}}$ and $\varepsilon_{\mathrm{m}}$ give the relative dielectric and metal permittivity, $\beta_{\mathrm{spp}}$ and $n_{\mathrm{eff}}$ are propagation constant and effective refractive index of SPPs, and $=2 \pi \lambda \mathrm{k}_{0}$ means wave number. Two-dimensional simulation is used to enhance sensor performance and achieve more accurate results and reduce simulation time. We must also choose the right height for the input wave, because too much wave height will reduce the wave penetration into the sensor structure and reduce the refractive index (can lead to longer propagation 
length) [26]. To describe the wave behavior, we draw a field distribution diagram (Fig.2). According to the figure, the wave goes to the resonators after starting to move. The resonant system is now considered as a single loop resonator whose effective radius is determined by the energy distribution. The middle cavity gets the largest share of the field distribution and creates a uniform state with the internal phase.

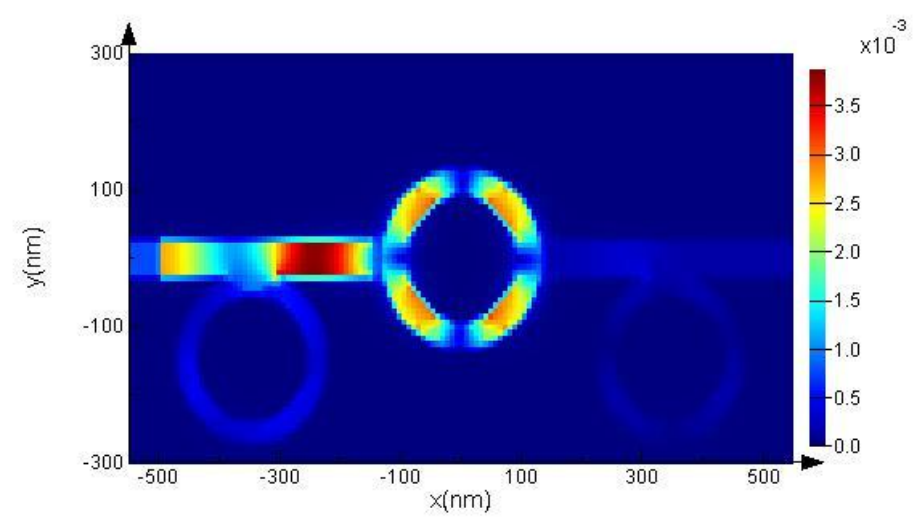

Fig.2. Electric field distribution at resonant frequency

MIM waveguide transmission spectra for different modes are plotted in Fig.3. When the middle ring is present in the sensor structure with one of the other three resonator $\left(R_{2}\right.$ ring or $R_{3}$ ring or cavity), the transmission spectrum has three peaks But when all four resonator $\left(\mathrm{R}_{1}\right.$ ring, $\mathrm{R}_{2} \operatorname{ring}, \mathrm{R}_{3}$ ring and cavity) are present in the sensor structure, the transmission spectrum has two peaks. Also, the wavelength change in this diagram ( $\mathrm{R}_{1}$ ring, $\mathrm{R}_{2}$ ring, $\mathrm{R}_{3}$ ring and cavity) is more and will reach a higher sensitivity factor. Therefore, we use the following equation to calculate the resonance conditions:

$\mathrm{N} \lambda=\operatorname{Re}\left(\mathrm{n}_{\text {eff }}\right) \mathrm{L}_{\text {eff }}, \mathrm{N}=1,2,3 \ldots \ldots$

Where $\mathrm{N}$ refers to mode number which is an integer. $\mathrm{n}_{\text {eff }}$ refers to the effective refractive index of the ring resonator which can be solved by Eq (6), Leff means effective perimeter, generally refers to the average of the inner and outer perimeters.

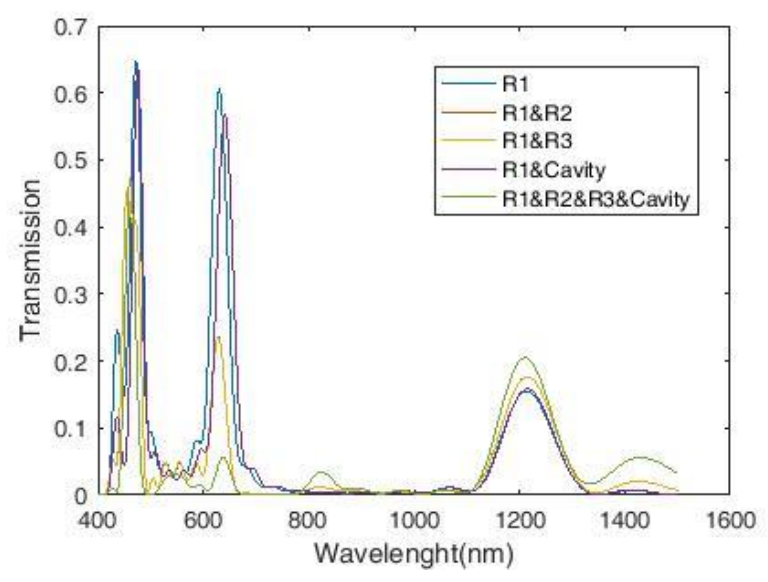

Fig.3. MIM waveguide transmission spectrum with resonators

\section{Fracture coefficient simulation and measurement methods}


The proposed plasmonic resonance behavior is investigated numerically and theoretically. For the numerical approach, we use the finite difference method of time domain with perfectly matched layer boundary conditions (PML) because this method effectively reduces numerical reflection. The uniform mesh sizes for the $\mathrm{x}$ and $\mathrm{y}$ directions are 8 and $8 \mathrm{~nm}$, respectively. The transmission line model is used to analyze the structure theory and the simulation is done in two dimensions, which is infinite in one dimension. We change the effective refractive index of the middle ring to evaluate the performance of the sensor. This change in refractive index is calculated in the wavelength range of 400 to $1500 \mathrm{~nm}$ and the transmission spectrum from the sensor device is shown in Fig.4. We increased the dielectric refractive index by a step of 0.01 from 1.15 to 1.2, which resulted in a change in the resonance spectra and wavelengths.

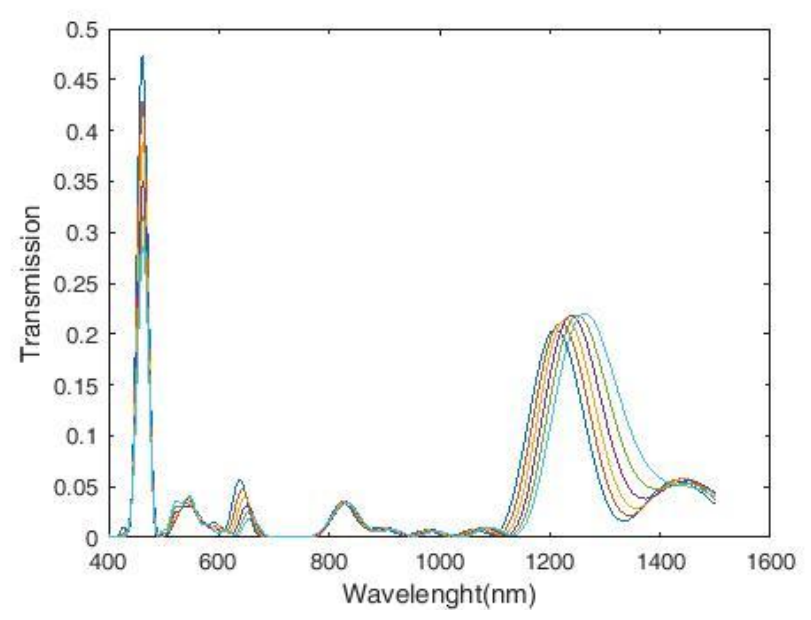

Fig.4. Transmission spectra of a plasmonic refractive index sensor

We increased the dielectric refractive index by a step of 0.01 from 1.15 to $1.2 \mathrm{~nm}$, which resulted in a change in the resonance spectra and wavelength. The first characteristic to be measured for a sensor is the $\mathrm{S}$ sensitivity, which is used to quantify the sensitivity of refractive index sensors:

$\mathrm{S}=\Delta \lambda / \Delta \mathrm{n} \quad(\mathrm{nm} / \mathrm{RIU})$

In this equation, $\Delta \lambda$ is the change in resonance wavelength and $\Delta \mathrm{n}$ is the change in refractive index. According to Fig.5, the sensor transmission spectra have two peaks, which according to Figure 4, have the highest sensitivity for the refractive index $\mathrm{n}=1.2$, which is equal to $1938 \mathrm{~nm} / \mathrm{RIU}$. According to this diagram, there is a relatively linear relationship between the two parameters of resonance wavelength and refractive index. 


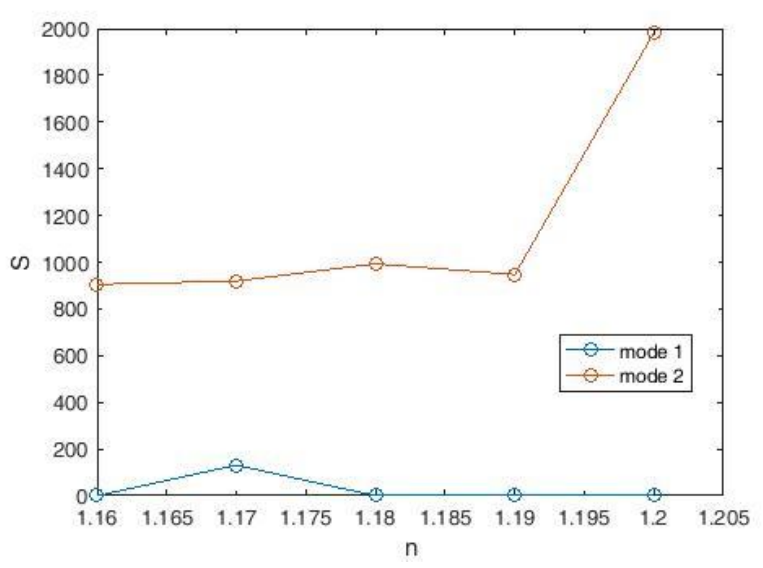

Fig.5. Plasmonic sensor sensitivity coefficient diagram

Because sensitivity alone is not a measure of good performance for comparing different types of sensors, and light resolution is also very important for sensors, figure of merit (FOM) is required to compare sensors:

$\mathrm{FOM}=\mathrm{S} / \mathrm{FWHM}$

(9)

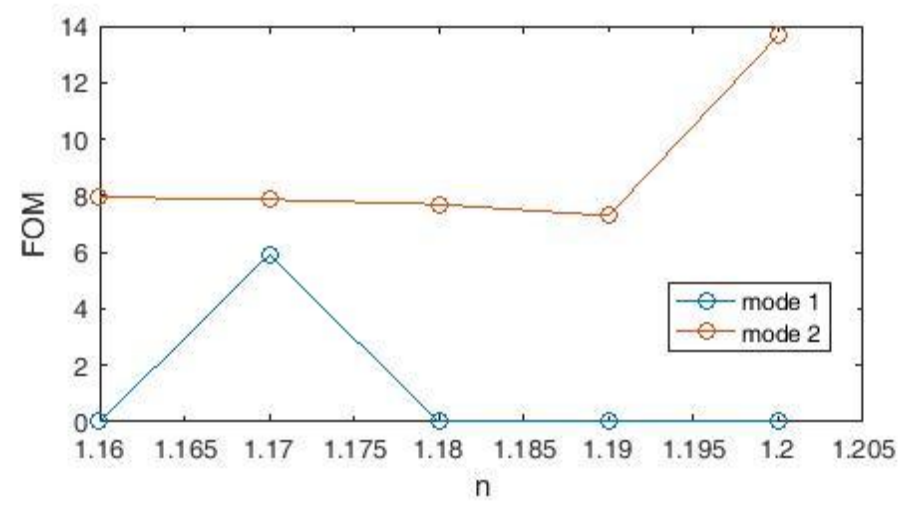

Fig.6. Plasmonic sensor FOM diagram

We also need the quality factor of the sensors:

$\mathrm{Q}=\lambda$ res / FWHM

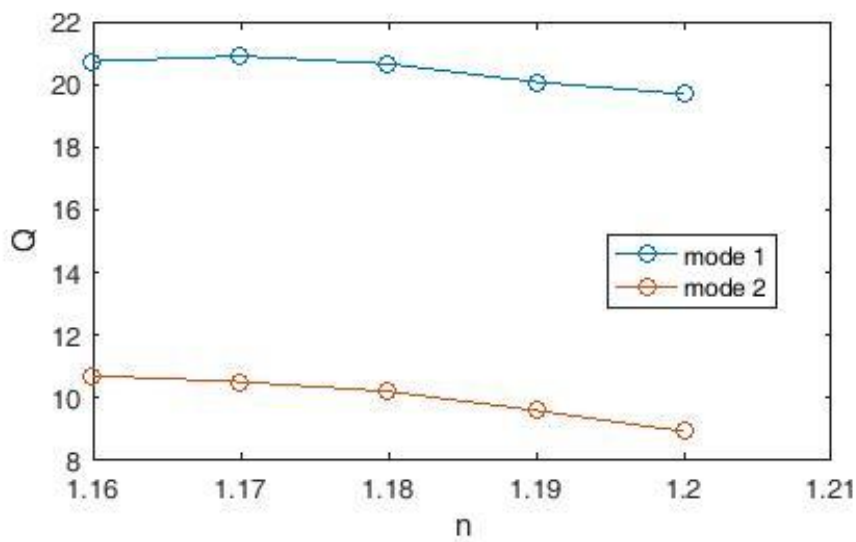


Fig.7. Plasmonic sensor quality coefficient diagram

According to Fig.7, the quality coefficient is obtained by using Equation 10 by dividing the wavelength by FWHM, whose value in the refractive index $n=1.2$, which has the highest sensitivity coefficient, reaches 8.934. Equations 8, 9 and 10 are the Measurement capabilities of plasmonic sensors obtained by changing the refractive index of the structure. Using these three equations, we plot the coefficients of sensitivity, quality, and figure of merit.

\section{Sensitivity analysis of the sensor by adding four teeth}

Now for better evaluation and to achieve a better quality sensor, we add four teeth with length $\mathrm{x}_{1}=\mathrm{x}_{3}$ $=20, \mathrm{x}_{2}=\mathrm{x}_{4}=40$, height $\mathrm{y}_{1}=\mathrm{y}_{3}=40$ and $\mathrm{y}_{2}=\mathrm{y}_{4}=20$, to the middle ring. The added teeth have an effect on the intensification of the middle ring, which changes the intensification wavelength or creates a new state. We change the refractive index of the middle ring and the refractive index of the other two rings and the teeth and the cavity and the two waveguides remain constant.

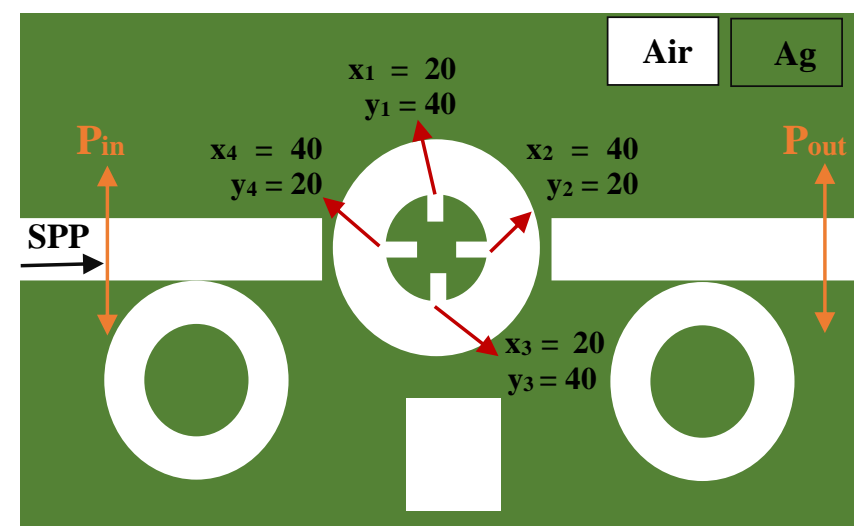

Fig.8. Sensitivity diagram of a plasmonic sensor with four teeth added

The transmission spectrum of the sensor device is shown in Fig.9. According to this diagram, there is a relatively linear relationship between the two parameters of resonant wavelength and refractive index, and the TM resonance in the range of 400 to $1500 \mathrm{~nm}$, gradually shifting Gives.

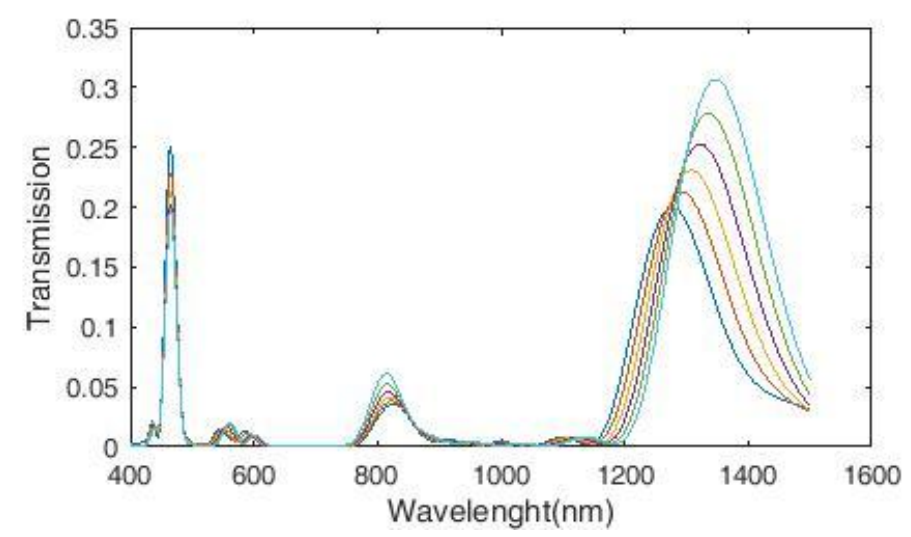

Fig.9. Transmission spectra of a plasmonic refractive index sensor 
By changing the refractive index by step 0.01 in the range 1.15 to 1.2 , we obtain the sensitivity coefficient of the new structure using Equation 8 and plot it (Fig.10).

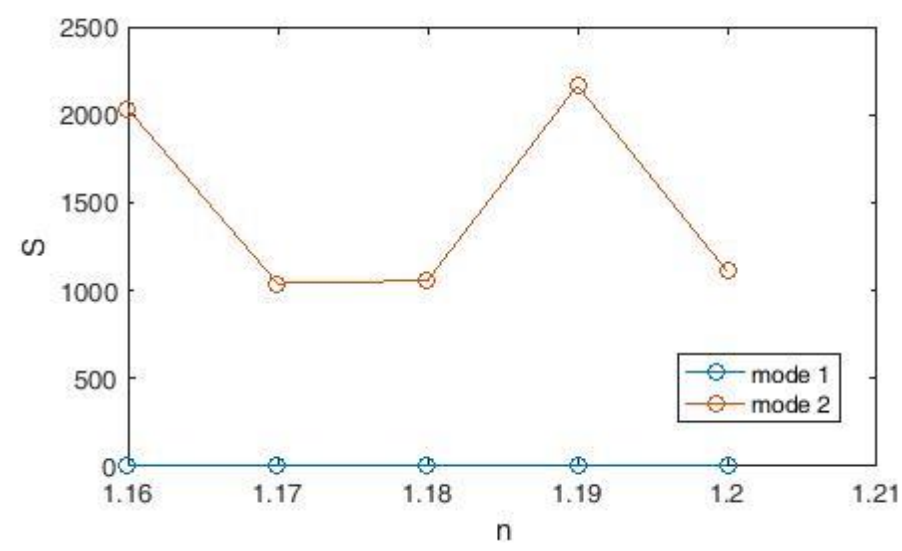

Fig.10. Plasmonic sensor sensitivity coefficient diagram

The refractive index diagram in Fig.10 examines the peaks in Fig.9. In the left peak, all sensitivity coefficients are equal to zero, but the right peak has good results and the highest sensitivity coefficient is equal to $2166 \mathrm{~nm} / \mathrm{RIU}$. The result is better than many similar articles. We now examine the figure of merit (FOM) of the designed sensor. Using Equation 9, we obtain its diagram and draw it.

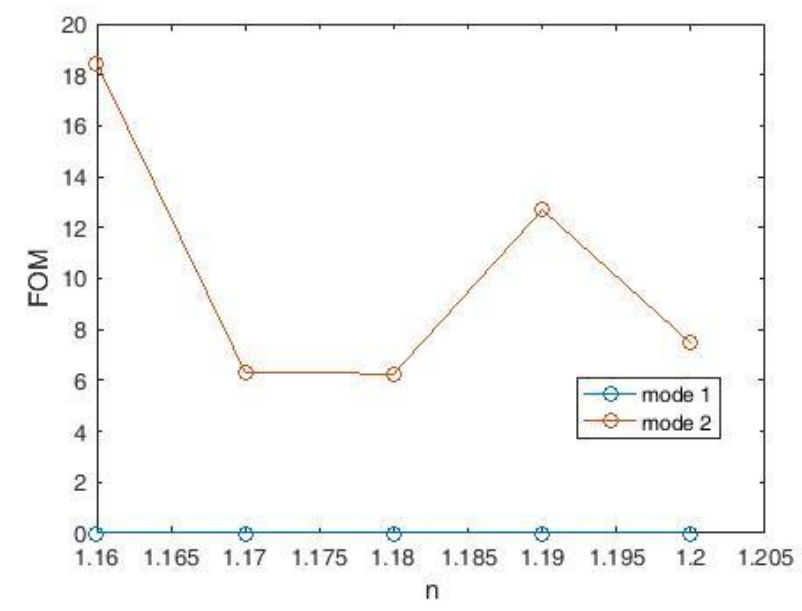

Fig.11. Plasmonic sensor FOM diagram

Like the sensitivity coefficient, all mode 1 numbers of the figure of merit (FOM) are zero, but mod 2 has better quality and the highest FOM value is 18.447. The next criterion for measuring the performance of this sensor is the quality factor $\mathrm{Q}$. 


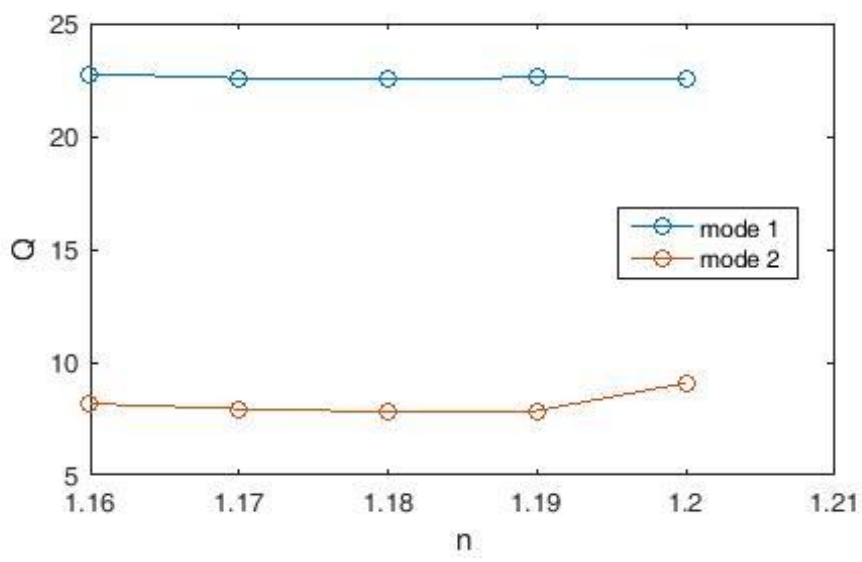

Fig.12. Plasmonic sensor quality coefficient diagram

The quality coefficient $\mathrm{Q}$ at $\mathrm{n}=1.16$ (which has the highest sensitivity coefficient) reaches $8.164 \mathrm{~nm}$.

\section{Bio-sensing}

Finally, we review an application for bioassay. The structure parameters remain constant. Since water is one of the most common solvents used in chemical and biological applications, it is necessary to test the performance of the sensor in water $(n=1.33)$ as presented in Fig.13. Since the concentration of the solution can be reflected by the refractive index, a set of different refractive index values around the water is used to measure the sensitivity and the figure of merit (FOM).

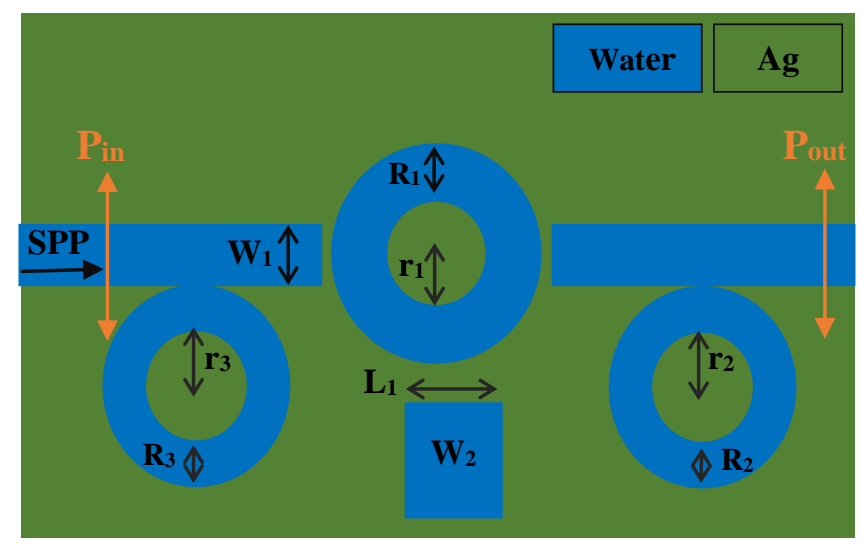

Fig.13. Two-dimensional image of a plasmonic sensor in water.

The transmission spectrum from the sensor device is shown in Fig.14. We have increased the refractive index of the middle cavity by a step of $0.0 \mathrm{~nm}$ from 1.34 to 1.38 . 


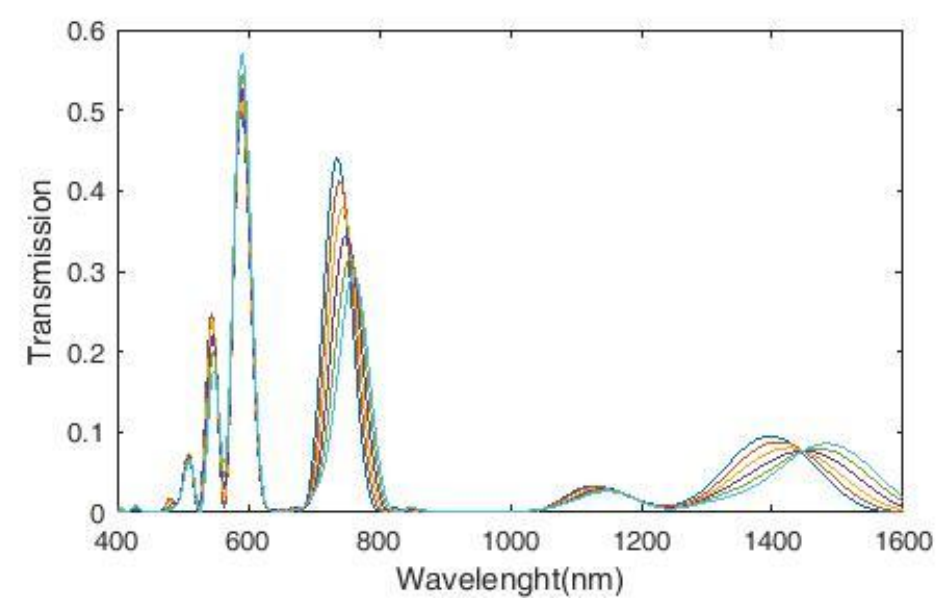

Fig.14. Transmission spectrum of a plasmonic sensor in water.

Using the transfer spectrum and using Equation 8, we calculate the sensitivity coefficient of the plasmonic sensor in water, which reaches the sensitivity coefficient of $2604 \mathrm{~nm} /$ RIU. This sensitivity coefficient is higher than similar tasks and proves that this designed sensor has high quality and good performance and also has a very good performance in water (Fig.15). Therefore, such structure can be a sensitive label-free and compact biosensor

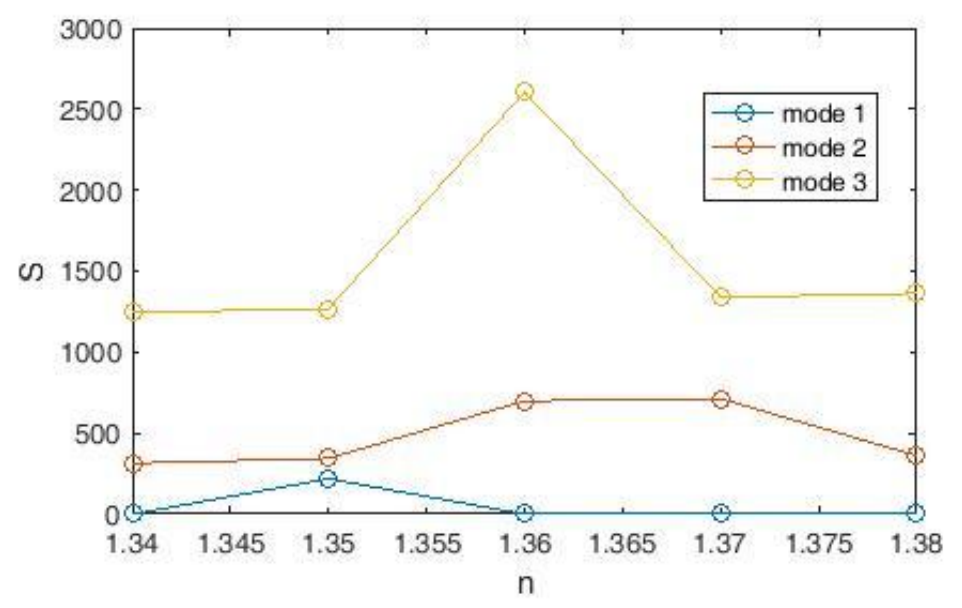

Fig.15. Plasmonic sensor sensitivity coefficient diagram in water

\section{Conclusion}

A new topology was proposed and analyzed to measure the refractive index. The proposed configuration has a high sensitivity of $2166 \mathrm{~nm} /$ RIU. This sensor is considered because a small change in the refractive index of the middle ring causes a significant change in the propagation characteristics of the wave. It also has a high sensitivity as a sensor in bioassay and has excellent performance with high optical resolution. The plasmonic sensor in water reaches a sensitivity factor of $2604 \mathrm{~nm} /$ RIU. Our results and physical analysis due to the small size of the structure and high sensitivity coefficient can cause excellent light control in plasmonic structures and also reduce losses.

Funding: This research received no external funding. 
Disclosures. The author declares no conflicts of interest.

Data Availability. No data were generated or analyzed in the presented research.

\section{Reference}

1. $\mathrm{Wu}, \mathrm{W}$. Ultra-high resolution filter and optical field modulator based on a surface plasmon polariton. Optics Letters 2016, 41.10, 2310.

2. K. Shiokawa, K. Yumoto, Y. Tanaka, H. Osaki, M. Sato, T. Kato, Y. Kato, M. Sera, Y. Ikegami, S.-I. Akasofu, K. Hayashi, T. Oguti, Y. Kiyam. Auroral Observations Using Automatic Optical Instruments: Relations with Multiple Pi 2 Magnetic Pulsations.1996.

3. Wu, D. Numerical study of an ultra-broadband near-perfect solar absorber in the visible and near-infrared region. Optics Letters 2017, 42.3, 450.

4. Yu, Y., Si J, Ning Y. Plasmonic wavelength splitter based on a metal-insulator-metal waveguide with a graded grating coupler. Optics Letters 2017, 42.2, 187.

5. Chen, L. Numerical analysis of a near-infrared plasmonic refractive index sensor with high figure of merit based on a fillet cavity. Optics Express 2016, 24.9, 9975

6. Cen C, Lin H, Huang J, Liang C, Chen X, Tang Y, Yi Z, Ye X, Liu J, Yi Y, Xiao S. (2018). A Tunable Plasmonic Refractive Index Sensor with Nanoring-Strip Graphene Arrays. Sensors.

7. Cheng F, Yang X, Gao J. (2014). Enhancing intensity and refractive index sensing capability with infrared plasmonic perfect absorbers. Opt Lett.

8. Cheng L, Wang Z, He X, Cao P. (2019). Plasmonic nanosensor based on multiple independently tunable Fano resonances. Beilstein J Nanotechnol.

9. Chou Chao CT, Chou Chau YF, Huang HJ, Kumara NTRN, Kooh MRR, Lim CM, Chiang HP. (2020). Highly Sensitive and Tunable Plasmonic Sensor Based on a Nanoring Resonator with Silver Nanorods. Nanomaterials (Basel).

10. E. Haque, S. Mahmuda, M. A. Hossain, N. H. Hai, Y. Namihira and F. Ahmed. (2019). "Highly Sensitive Dual-Core PCF Based Plasmonic Refractive Index Sensor for Low Refractive Index Detection," in IEEE Photonics Journal.

11. El Shamy RS, Khalil D, Swillam MA. (2020). Mid Infrared Optical Gas Sensor Using Plasmonic Mach-Zehnder Interferometer. Sci Rep.

12. Gryga, M.; Ciprian, D.; Hlubina, P. (2020). Bloch Surface Wave Resonance Based Sensors as an Alternative to Surface Plasmon Resonance Sensors. Sensors.

13. Gomez-Cardona, N.; Reyes-Vera, E.; Torres, P. (2020). High Sensitivity Refractive Index Sensor Based on the Excitation of Long-Range Surface Plasmon Polaritons in H-Shaped Optical Fiber. Sensors.

14. Tong, L. Recent advances in plasmonic sensors. Sensors 2014, 14.5, 7959.

15. Maier, Stefan A. Plasmonics: Fundamentals and Applications. Springer Berlin 2014, 52.11, 49-74.

16. Shu, Changgan. The sensing characteristics of plasmonic waveguide with a ring resonator. Optics Express 2014, 22.7, 7669-77.

17. Yan, Shu Bin. A Refractive Index Sensor Based on a Metal-Insulator-Metal Waveguide-Coupled Ring Resonator. Sensors 2015, 15.11, 29183-91.

18. Huang, Ye Xiong. A plasmonic refractive index sensor based on a MIM waveguide with a side-coupled nanodisk resonator. IEEE, International Conference on Embedded and Real-Time Computing Systems and Applications IEEE 2014, $1-5$.

19. Wu, Tiesheng. A nanometeric temperature sensor based on plasmonic waveguide with an ethanol-sealed rectangular cavity. Optics Communications 2015, 339, 1-6.

20. Tang, Y. Refractive Index Sensor Based on Fano Resonances in Metal-Insulator-Metal Waveguides Coupled with Resonators. Sensors 2017, 17.4, 784.

21 Zhang, Zhidong. Fano Resonance Based on Metal-Insulator-Metal Waveguide-Coupled Double Rectangular Cavities for Plasmonic Nanosensors. Sensors 2016, 16.5, 642.

22. Zafar, Rukhsar, and M. Salim. Enhanced Figure of Merit in Fano Resonance-Based Plasmonic Refractive Index Sensor. IEEE Sensors Journal 2015, 15.11, 6313-6317.

23. Zhao, X., Z. Zhang, and S. Yan. Tunable Fano Resonance in Asymmetric MIM Waveguide Structure. Sensors 2017, 17, 7 .

24. Chen, Jianjun. Coupled-Resonator-Induced Fano Resonances for Plasmonic Sensing with Ultra-High Figure of Merits. Plasmonics 2013, 8.4, 1627-1631.

25. Khurgin, J. B. How to face the loss in plasmonics and metamaterials. Nature Nanotechnology 2014, 10(1), 2-6.

26. Veronis, G., \& Fan, S. Modes of subwavelength plasmonic slot waveguides. Journal of Lightwave Technology 2007, 25(9), 2511-2521. 